\title{
RECIPROCAL TRANSFER OF BLASTOCYSTS BETWEEN THE RAT AND RABBIT
}

\author{
K. YOSHINAGA* $\uparrow$ AND G. E. ADAMS \\ A.R.C. Unit of Reproductive Physiology and Biochemistry, \\ University of Cambridge
}

(Received 24th February 1967)

\begin{abstract}
Summary. Blastocysts were transferred reciprocally between the rabbit and rat. Rat blastocysts either with or without the zona pellucida survived transfer to the oviduct of the pseudopregnant rabbit for $48 \mathrm{hr}$, as demonstrated by their capacity to implant upon re-transfer to rats; however, only a few survived to term. Rabbit blastocysts degenerated when transferred for $48 \mathrm{hr}$ to the uterus of rats in the 'delayed implantation' condition.
\end{abstract}

Mammalian eggs, especially at the morula stage, may undergo development following transfer to the genital tract of another species but, with certain exceptions, notably in transfers between sheep and goats (Warwick \& Berry, 1949; Lopyrin, Loginova \& Karpov, 1951) and rats and mice (Tarkowski, 1962), they do not survive to implantation, mostly degenerating at the blastocyst stage. Interspecies transfer of blastocysts has been reported from the rat to the mouse (Tarkowski, 1962) and reciprocally between the rabbit and ferret (Chang, 1966). The only report on the re-transfer of blastocysts to recipients of their own species after incubation in an alien environment is that of Tarkowski (1962) who found that their development was impaired. Recently, we have examined the survival of blastocysts following transfer between a species showing delayed implantation and one that does not, namely rat or rabbit.

Blastocysts with the zona pellucida still present were collected from nineteen rats on the morning of Day 5 of pregnancy (Day $1=$ day spermatozoa are found in vagina). Zona-free blastocysts were obtained from twenty-five rats 7 to 11 days after mating, which had been spayed on Day 3 and then treated daily with $2 \mathrm{mg}$ progesterone for 4 to 8 days.

The two types of rat blastocyst, totalling 229 and 192 respectively, were transferred to the ligated oviducts of twenty-six rabbits injected i.v. $72 \mathrm{hr}$ earlier with 25 i.u. HCG (Lutormone, Burroughs Wellcome) to induce ovulation. After $48 \mathrm{hr}$ the rabbits were killed to permit recovery of the rat blastocysts, some of which were re-transferred to the uteri of recipient rats on the

* Lalor Foundation Fellow, 1965-66.

† Present address: Worcester Foundation for Experimental Biology, Shrewsbury, Massachusetts 01545 , U.S.A.

$\ddagger$ Postal address: 307 Huntingdon Road, Cambridge. 
5 th day of pseudopregnancy. These recipients were later examined twice by laparotomy, 4 and 9 days after transfer, to record the number of implantations and foetuses: those maintaining the pregnancy were either killed near term or allowed to deliver their young.

Forty-seven blastocysts collected from seven rabbits $96 \mathrm{hr}$ p.c. were transferred to the uteri of ten rats which had been spayed on Day 3 of pseudopregnancy and then treated daily for 4 to 7 days with $2 \mathrm{mg}$ progesterone. After $48 \mathrm{hr}$, the rabbit blastocysts were recovered for examination. Physiological saline was used as the recovery and transfer medium in every case.

Of the 229 rat blastocysts transferred to thirteen rabbits, seventy-eight $(34 \cdot 1 \%)$ were recovered after $48 \mathrm{hr}$, including fourteen which had escaped from the zona pellucida (Table 1). No regular mucin layer had accumulated on the blastocysts. Upon re-transfer to synchronized recipient rats forty-seven blastocysts, which still possessed the zona pellucida, gave rise to twenty-two implantations, but only three of these survived to Day 14, and two to term. Twelve of the zona-free blastocysts were transferred to three rats, and six implants were later observed in one recipient which subsequently gave birth to two young.

One hundred and ninety-two 'zona-free' blastocysts were transferred to thirteen rabbits, nine of which yielded thirty-nine (20.3\%) blastocysts $48 \mathrm{hr}$ later. Thirty-six of these blastocysts, all of which appeared healthy, were re-transferred to eleven recipient rats, in eight of which twenty-three implantations were subsequently recorded. However, only four of the implants survived to Day 14 and two to term.

The results are summarized in Table 1, which shows that rat blastocysts are able to survive in the rabbit oviduct for at least $48 \mathrm{hr}$, as demonstrated by their capacity to implant upon re-transfer to synchronized recipient rats and, in some cases, even to develop to term. However, most of the implants showed only a very limited capacity to survive. This mortality is reminiscent of Tarkowski's (1962) finding that the development of rat blastocysts 'incubated' in mice was impaired. After the re-transfer of fifty-one blastocysts to six recipient rats, he recorded nineteen decidual swellings but only two contained normal embryos. The blastocysts had given rise to hollow 'trophoblastic vesicles', which apparently 'did not differ from normal ones in their ability to elicit typical decidual reactions'.

By comparing his own results with those of Dickmann \& Noyes (1960) and Noyes \& Dickmann (1961), Tarkowski concluded that the age of the blastocysts could not itself be responsible for the mortality and that it must be caused by prolonged development in the mouse oviduct. Our own experience supports Tarkowski's conclusion, though we consider that a 'dormant' blastocyst may be equally affected by an alien environment. In fact, blastocysts obtained from rats undergoing delayed implantation fared no better than those taken from a normal pregnancy.

Degeneration of rabbit blastocysts in the uterus of recipient rats undergoing 'delayed implantation' indicates that this condition, though conducive to the extended survival of native blastocysts, was unfavourable for those of the rabbit. Chang (1966) also found that rabbit blastocysts degenerated in the 


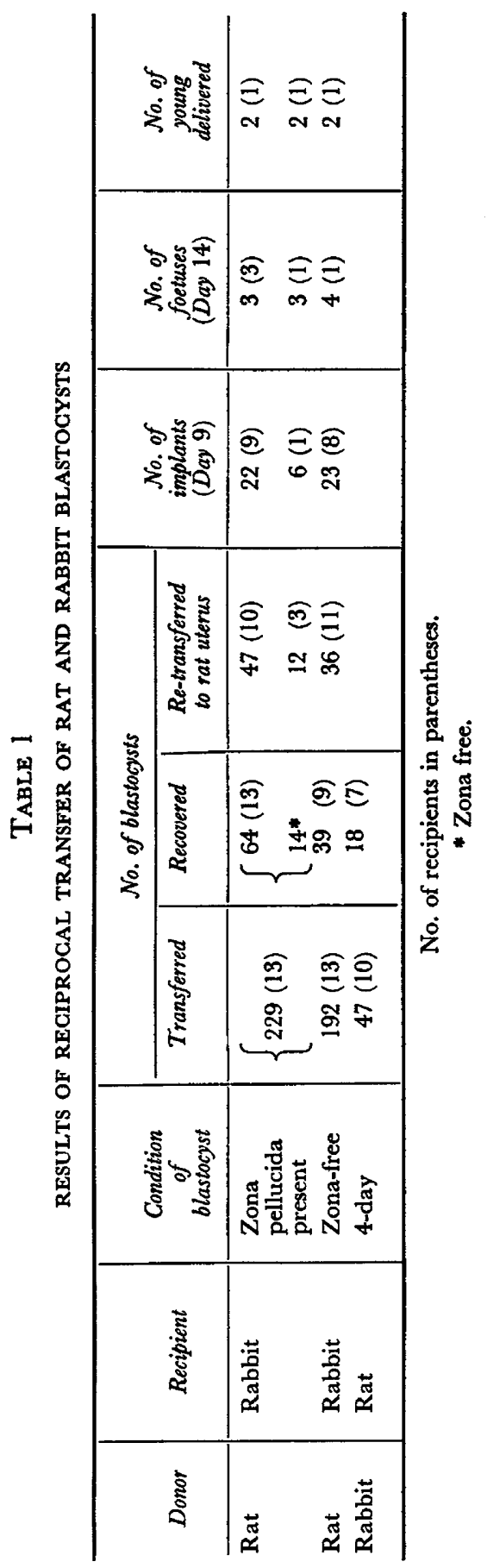


uteri of pseudopregnant ferrets. With possible exceptions, it appears that, whereas eggs in cleavage stages may survive and develop for various periods of time in another species, blastocysts are much more sensitive, either degenerating quickly, as do those of the rabbit or sheep, or suffering some degree of incapacitating damage.

So far no experimental situation has been discovered in which the blastocysts of species not normally known to show delayed implantation can be made to undergo this process. It appears that a high degree of specialization of the blastocyst and its environment is necessary for successful achievement of this state.

One of the authors (K.Y.) wishes to acknowledge his indebtedness to the Lalor Foundation for financial support.

\section{REFERENCES}

Chang, M. C. (1966) Reciprocal transplantation of eggs between rabbit and ferret. F. exp. Zool. 161, 297.

Dickmann, Z. \& Noyes, R. W. (1960) The fate of ova transferred into the uterus of the rat. F. Reprod. Fert. 1, 197.

Lopyrin, A. I., Logrnova, N. V. \& Karpov, P. L. (1951) The effect of changed conditions during embryogenesis on growth and development of lambs. Sov. Zootekh. 6, 83 (in Anim. Breed. Abstr., 1952, 20, No. 729).

Noyes, R. W. \& Dickmann, Z. (1961) Survival of ova transferred into the oviduct of the rat. Fert. Steril. 12, 67.

TARKowskI, A. K. (1962) Inter-specific transfers of eggs between rat and mouse. 7. Embryol. exp. Morph. 10, 476.

WARWICK, B. L. \& BERRY, R. O. (1949) Inter-generic and intra-specific embryo transfers in sheep and goats. F. Hered. 40, 297. 